\title{
Metal/Organic Interface Analysis Based on Photoelectron Spectroscopy and its Application Studies on the Next Generation Electronic Devices
}

\author{
Jouhahn Lee \\ Korea Basic Science Institute, Jeonju Center, Korea \\ jouhahn@kbsi.re.kr
}

(Received: January 15, 2009; Accepted: February 17, 2009)

\begin{abstract}
Recently there is a rapid growth of the R\&D of organic thin film and molecular-scale electronics. Most of the presently feasible applications use the structures of thin films, in which multilayer of organic material is sandwiched between metal or oxide electrodes. Since device functions come from the charge transport in these devices, the electronic structure of such materials in the bulk, in the film, and at the interfaces plays essential role in the organic nano-device performances. The study of such interfaces needs the combination of various experimental and theoretical methods, in which the role of x-ray photoelectron spectroscopy (XPS) and ultraviolet photoelectron spectroscopy (UPS) becomes most powerful techniques to explore those physical and chemical characterizations. In this presentation, the analytical techniques using electron spectroscopy techniques such as UPS (Ultraviolet Photoelectron Spectroscopy)/ XPS (X-ray photoelectron spectroscopy) with NEXAFS (Near Edge X-ray Absorption Fine Structure) will be mainly presented. These analytical techniques have provided the correlation between the electronic structure and the performance along with the improvement of device efficiency. In addition, another latest surface analysis technique such as Kelvin Probe Microscopy and SPEM(Scanning Photoelectron
\end{abstract}

Microscopy) will be also introduced. These analytical approaches for OLED should provide the essential information on fundamental understanding for Metal/Organic interface and the degradation mechanism of organic nano-devices.

With photoelectron spectroscopy studies, the electronic structure changes occurring at the interface directly indicates the performances of organic devices. Therefore, the charge transport through the whole organic device can be explained by applying photoemission analysis tools. For example, $\mathrm{Al} / \mathrm{LiF} / \mathrm{Alq} 3$ and $\mathrm{Al} / \mathrm{CaF} 2 / \mathrm{Alq} 3$, the most commonly used OLED cathode structures were probed with the conventional XPS and UPS with in situ thin film preparation process. These results revealed that the lowering the barrier injection at the interface by dipole formation has caused the drastic improvement of device performances. Figure 1 shows the electronic structure changes at the interface as a function of deposited thin film. Figure 2 shows the device performance in connection with the results from the figure 1 . In this case, the correlation between the performance and the electronic structure change is clearly seen; Band shift or HOMO level shift causes lowering the injection barrier, which eventually induce the high performance. 


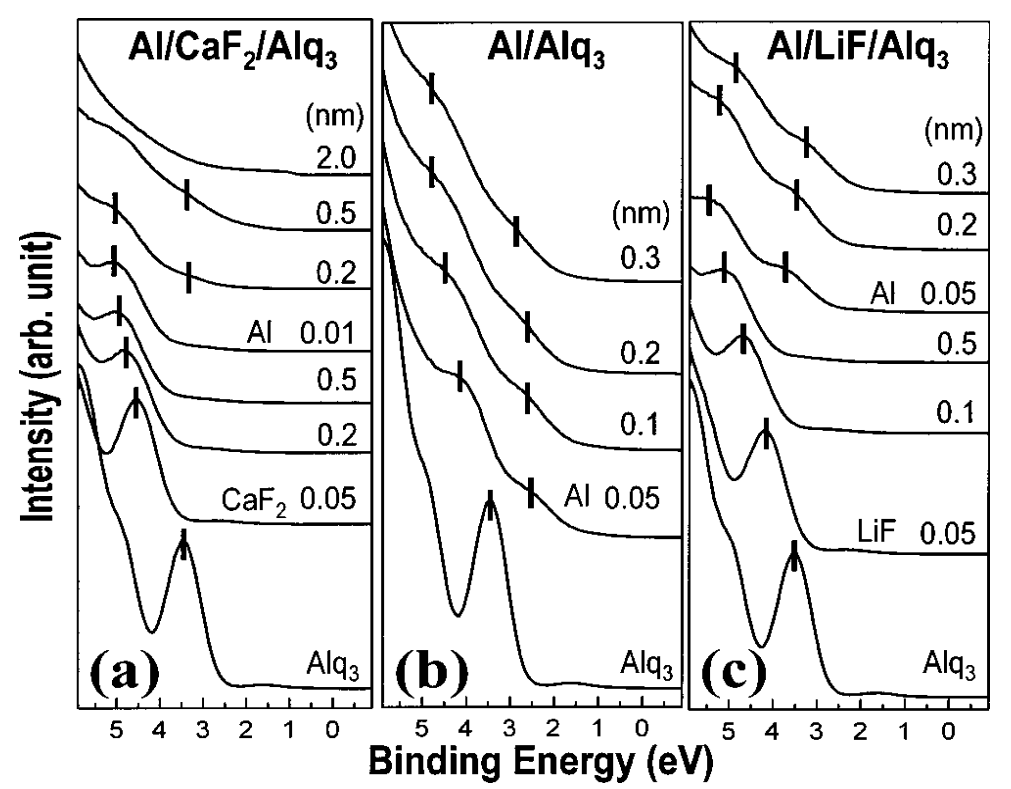

Fig. 1 (a) The evolution of the valence band spectra for $\mathrm{Al} / \mathrm{CaF}_{2} / \mathrm{Alq}_{3}$ in interface with increasing $\mathrm{CaF}_{2}$ and Al coverage. Similar spectra for Al-only (b) and Al/LiF (c) cathodes are also shown. The vertical bars indicate HOMO position and gap states.

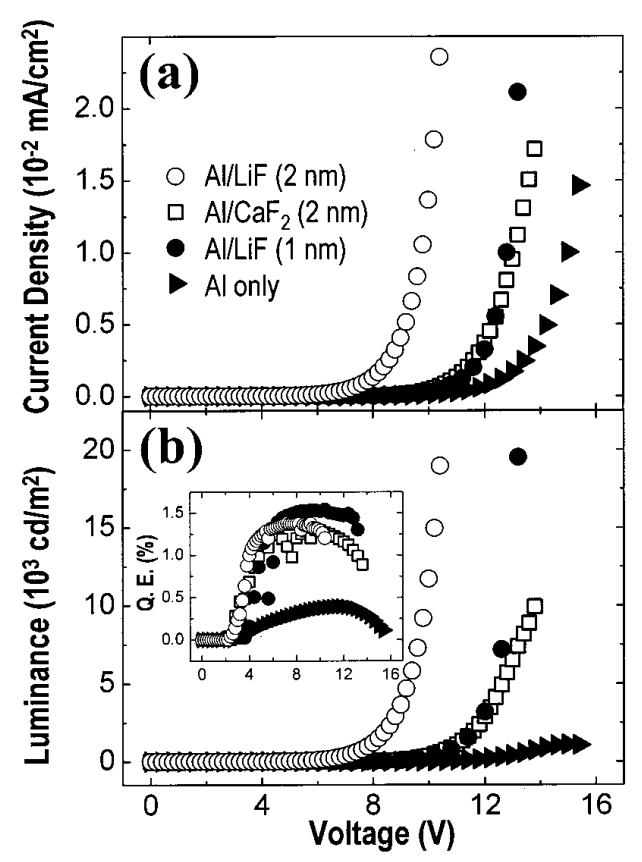

Fig. 2 (a) $I-V$ and (b) $L-V$ curves of the devices with $\mathrm{Al} / \mathrm{LiF} / \mathrm{Alq}_{3}, \mathrm{Al} / \mathrm{CaF}_{2} / \mathrm{Alq}_{3}$, and $\mathrm{Al} / \mathrm{Alq}_{3}$ interfaces. The inset of (b) shows the EQE of the same devices. 